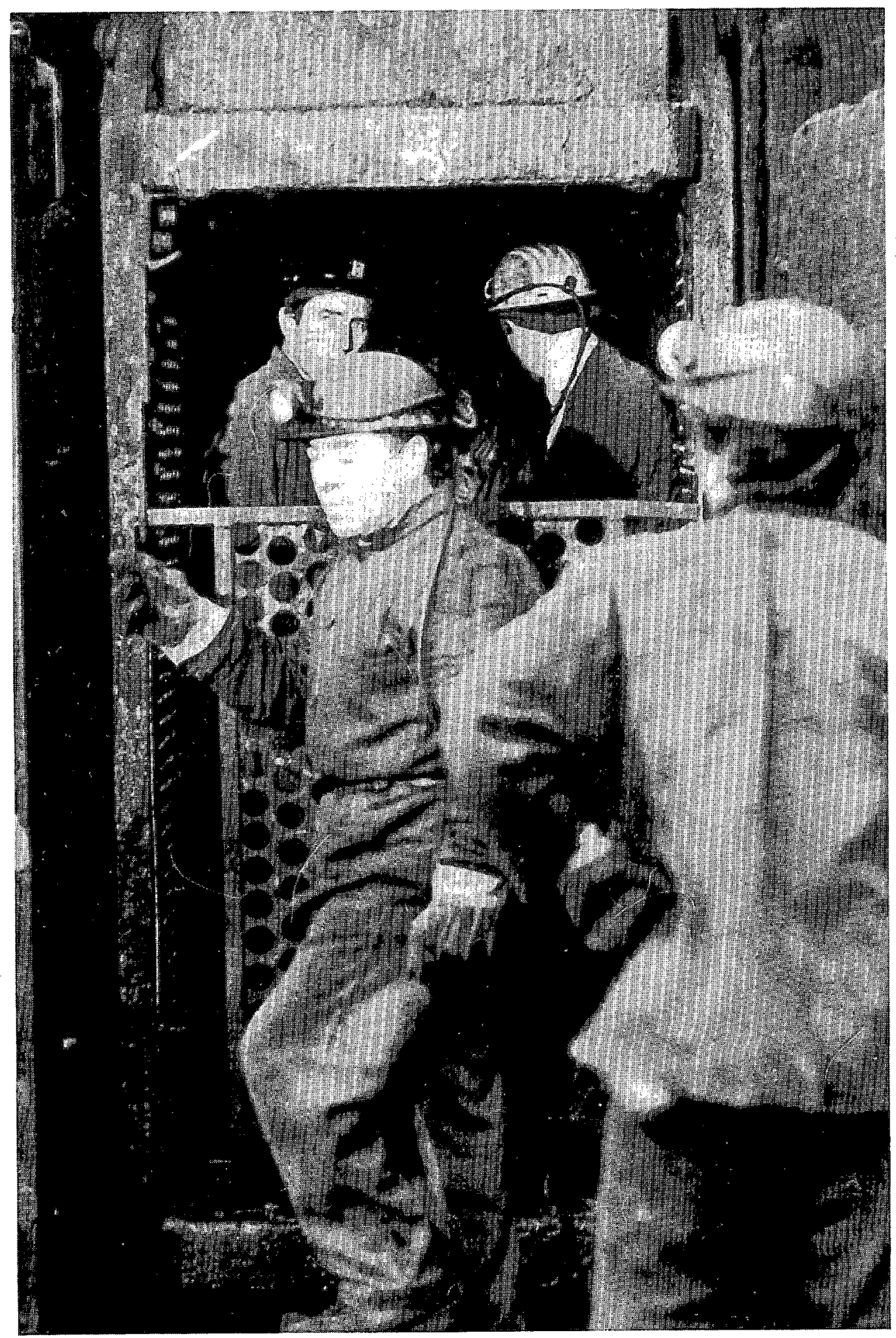




\title{
Ambiguités et contradictions des rapports entre syndicats et étrangers: le cas des Italiens
}

\author{
Anne Morelli, historienne et déléguée \\ syndicale CGSP au conseil d'entreprise de l'ULB
}

\section{Avant 1918}

On sait que la Belgique a été généralement une terre d'émigration et non d'immigration jusqu'àla fin de la première guerre mondiale.

Jusqu'en 1918 les Belges sont plus nombreux à tenter fortune à l'extérieur que les étrangers à venir s'installer ici. Ces étrangers existent cependant. Il $y$ a parmi eux beaucoup de frontaliers mais aussi - déjà - des Polonais employés dans les mines et des Italiens recrutés pour des chantiers. Leur présence en Belgique n'a évidemment d'intérêt pour le patronat que dans la mesure où ils sont "moins exigeants" que les autochtones du point de vue salarial et des conditions de travail. C'est ainsi qu'on peut expliquer à la fin du 19ème siècle la présence d'Italiens sur un chantier de construction de chemin de fer à Vonêche (Namur) et l'animosité à leur égard des ouvriers locaux qui tentent d'éliminer ces "gâcheurs de métier" justifiant ainsi l'établissement d'un poste de gendarmerie pour les "protéger". Les Polonais sont bien appelés dans les mines comme briseurs de grève, même s'il s'avère que certains - malgré l'isolement dans lequel on les tient - refuseront ce rôle de "jaunes" et se solidariseront des revendications des ouvriers belges.

\section{L'entre-deux-guerres}

La stratégie patronale reste semblable dans l'entre-deux-guerres. Les mines de Wallonie voient encore affluer des ouvriers peu exigeants venus des campagnes flamandes (les trains de navetteurs leur permettent d'entretenir leurs petits lopins et donc de se contenter d'un salaire d'appoint) mais aussi des Italiens du Nord, des Polonais et des Africains du Nord.

Rapidement, on peut se rendre compte qu'une partie de cette main-d'oeuvre importée pour sa docilité est en fait plus politisée qu'on ne le croit et rejoint les revendications de la main-d'oeuvre locale. Ainsi, lors d'une manifestation de grévistes dans le Borinage en 1924, on peut voir des Algériens et des Marocains coiffés de leur chéchia rouge, porter un calicot de solidarité avec les revendications des mineurs borains ${ }^{(1)}$ tandis que parmi les immigrés polonais et italiens, en principe uniquement "économiques", il y a aussi des communistes qui vont tenter d'organiser les revendications de leurs compatriotes.

Les syndicats catholiques des Francs-Mineurs organisent depuis 1923 des sections polonaises ayant leur propre journal mais en-dehors des Belges, dans des sections distinctes ${ }^{(2)}$. 
La préoccupation des problèmes de migrants stagne dans les syndicats chrétiens au niveau de l'abstraction et ne se traduit pas dans le concret. La Confédération Internationale des Syndicats chrétiens par exemple, demande l'égalité en matière de législation spéciale pour les immigrés et le maintien de leurs droits acquis avant l'émigration mais cette revendication ne s'inscrit pas dans la réalité belge ${ }^{(3)}$. L'hebdomadaire CSC, par exemple, n'envisage pas le problème des migrants dans la pratique ${ }^{(4)}$.

Malgté les menaces d'expulsion qui pèsent sur eux, des Polonais de gauche entament aussi chez leurs compatriotes un travail d'organisation syndicale.

Dans le secteur minier, deux syndicats de gauche occupent le terrain, la Centrale des Mineurs (branche de la Commission syndicale belge, socialiste) et les Chevaliers du Travail (qui adhère à l'Internationale des Syndicats rouges, communiste) en perte de vitesse qui seront relayés par la Centrale révolutionnaire des Mineurs.

Les immigrés italiens de l'entre-deux-guerres, au nombre de 30.000 , sont majoritairement antifascistes et largement sympathisants du parti communiste italien. Ils accusent régulièrement les dirigeants de la Commission syndicale belge d'être xenophobes ${ }^{(5)}$, de mener, auprès des métallurgistes comme des mineurs belges, campagne contre les ouvriers étrangers ${ }^{(6)}$ et de ne jamais présenter les revendications particulières aux immigrés au cours des luttes ouvrières ${ }^{(7)}$.

Le mémoire de FrankCaestecker ${ }^{(8)}$ a montré avec éloquence le peu de solidarité que les étrangers ont eu à attendre des syndicats belges, surtout en période de crise. La solidarité qu'ils proclament est essentiellement verbale et jusqu'à la moitié des années trente le syndicalisme socialiste joue la carte - très populaire - de la xénophobie. Ce n'est qu'à partir de 1937 qu'il change d'orientation et appuie davantage les étrangers.

Officiellement, la presse du syndicat socialiste dénonce pourtant le fascisme et se déclare solidaire des "pauvres Italiens exilés"
Le mouvement syndical belge, par exemple, publie les appels de la CGT italienne ${ }^{(10)}$ et ouvre fréquemment ses colonnes à des proscrits italiens. Cependant, sur le terrain, la Centrale des mineurs, dépendant de la Commission syndicale socialiste, a pour revendication, lors de la grève de 1932, le tenvoi des mineurs étrangers, revendication qui ne sera d'ailleurs pas satisfaite par les patrons ${ }^{(11)}$. En général, la position de la Commission syndicale est caractérisée par une attitude double : d'une part, elle est protectionniste et combat le recrutement des travailleurs étrangers pour l'industrie belge ${ }^{(12)}$ et d'autre part, elle assure qu'elle défend les étrangers installés en Belgique que ce soit au point de vue de leurs conditions de vie matérielle ou au point de vue de leur liberté d'expression ${ }^{(13)}$.

Pierre Van Maldere, qui défend le plus souvent la position du syndicat socialiste sur la question des immigrés ${ }^{(14)}$, est tout à fait représentatif de ce dualisme. Il demande que les étrangers soient traités à égalité avec les Belges mais véhiculeà leur sujet les pires stéréotypes.

"L'ouvrier étranger immigré mène une vie animale; il est à peu près satisfait lorsque avec son salaire il a pu se loger, se vêtir, se nourrir (encore conviendrait-il de voir de près comment il est logé, vêtu et nourri); il lit peu ou pas (beaucoup, d'ailleurs, sont des illetrés) et est en général insensible aux manifestations de l'esprit, comme du reste, aux spectacles d'art que, parfois, pour ses loisirs, on lui offre, mais qu'il dédaigne avec un souverain mépris [...]. Les ouvriers étrangers dans leur ensemble, se distinguent de nos compatriotes par la médiocrité de leur existence matérielle et l'absence de toute préoccupation morale élevée" (15).

Pour le cas particulier des Italiens, P. Van Maldere concède que certains, "bons pères de famille, prévoyants, rangés, occupent leurs loisirs à la lecture, à la promenade. Mais c'est là l'exception [...]. Certains groupes d'Italiens ont des moeurs déplorables; ils sont sales, mal nettoyés, mal habillés et d'aucuns vivent dans une promiscuité repoussante." (15) 
Les articles d'E. Bastiaenen ${ }^{(17)}$, Jef Rens ${ }^{(18)}$, Corn. Mertens ${ }^{(19)}$ et même Paul M.G. Levy ${ }^{(20)}$, parus dans Le mouvement syndical belge sur le problème des étrangers en Belgique s'écartent peu de ce schéma.

C. Mertens, par exemple, plaide pour une égalité des salaires et des conditions de travail mais il veut l'assimilation complète et rapide des étrangers auxquels il reproche - très contradictoirement avec le reproche qui leur est formulé de se contenter de bas salaires - de ne penser qu'à "amasser de l'argent en prévision d'un retour éventuel dans leur pays d'origine munis de quelques économies." (21)

Sur pression des milieux antifascistes italiens, les carriers, les mineurs, les ouvriers du bâtiment italiens adhèrent assez largement aux syndicats mais la tentative des syndicats socialistes belges de les faire "encadrer" par un permanent sera un échec cuisant. En effet, Silvio Barro, venu à cet effet de Paris, est un socialiste qui situe son activité dans le cadre de la lutte anticommuniste au sein de la Commission syndicale et qui tente d'évincer les communistes italiens en vertu de la motion Mertens votée par les socialistes belges. Cette motion visait à exclure les communistes des organes dirigeants du syndicat.

Or, ce que les socialistes belges ont mal perçu c'est que le rapport de force dans l'émigration italienne est nettement favorable aux communistes. Sans leur appui, il est impossible de syndiquer massivement les Italiens.

La mission de Barro sera donc une mission impossible et après un an et demi de tentatives avortées, Barro regagne Paris et la Commission syndicale belge décide de ne pas le remplacer ${ }^{(22)}$.

$\mathrm{Si}$ les syndicats belges dans l'entre-deux-guerres se "désintéressent" des étrangers, est-ce comme le suggèrent certains, une stratégie?

Abandonnés par le syndicat, les étrangers se feraient alors briseurs de grèves. En cas d'échec des grèves qu'ils patronnent, lessocialistes pourraient ainsi faire retomber la responsabilité de l'échec sur les étrangers et entamer une campagne xéno- phobe - très populaire - qui ferait oublier leur propre rôle ${ }^{(23)}$.

Cecynismen'a sans doute pas été de mise et il faut plutôt retenir d'une part, les campagnes de mises à l'écart des "gauchistes" - à plus forte raison étrangers - dans le syndicat socialiste, mais aussi des facteurs objectifs extérieurs au syndicat. Les syndicalistes étrangers sont susceptibles de renvoi de la part de leur patron et d'expulsion de la part de la Sûreté. Dans ce domaine, la législation officielle sur la liberté d'association et la pratique de la police des étrangers sont en flagrante et continuelle opposition.

En outre, les étrangers dépendent souvent de leur logeur, et les cantiniers, pour beaucoup liés au patronat, interdisent l'accès aux dortoirs des étrangers aux propagandistes du syndicat ${ }^{(24)}$.

L'action antisyndicale des prêtres polonais et italiens et des fascistes ou l'obstacle de la langue sont d'autres empêchements à la syndicalisation massive des étrangers. Mais c'est l'attitude des syndicats à l'encontre des grévistes étrangers lors des grèves minières de l'entre-deux-guerres qui a sûrement été l'élément le plus déterminant dans la désaffection étrangère vis-à-vis des syndicats. Pour les Italiens, ils avaient commencé à affluer dans les syndicats belges en 1924, sur un appel très ferme du PCI en revendiquant pour les ouvriers étrangers le droit de coalition et de grève, des salaires égaux et des logements salubres ${ }^{(25)}$. Le PCI lançait régulièrement des campagnes de propagande parmi les immigrés pour le recrutement syndical et la solidarité avec le mouvement ouvrier belge.

Lors des grèves, au risque d'être expulsés, de nombreux étrangers n'avaient pas manqué de s'y associer $^{(26)}$ mais une grande déception allait les attendre.

En effet, bien que les étrangers cotisent au syndicat belge au même titre que les autres adhérents, ils sont exclus du bénéfice des indemnités de chômage et de grève versées par les syndicats. Une réunion houleuse a lieu à ce sujet en 1930 à la Maison du Peuple de Bruxelles. Les dirigeants de 


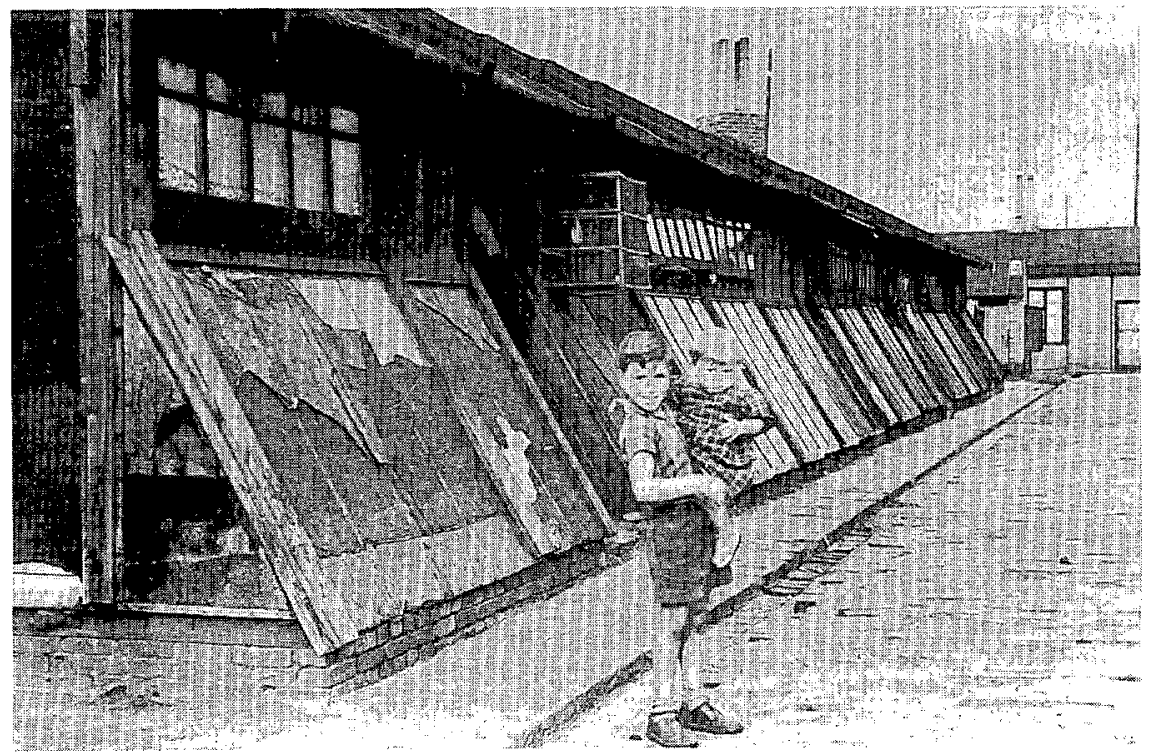

Barakken van

Italiaanse mijnwerkers in

Winterslag, 1954

la Commission syndicale promettent de se pencher sur le problème et un conseil de sept membres est élu pour le résoudre ${ }^{(27)}$.

Rien ne s'arrange cependant et; malgré les belles promesses d'un sérieux examen de ces propositions (28), lors des grèves de mineurs de juillet 1932, une discrimination totale est perpétrée par la Commission syndicale qui indemnise les seuls grévistes de nationalité belge!

Un comité syndical italien présente en décembre 1932 les revendications des Italiens au secrétaire du comité fédéral des syndicats bruxellois. Aucune suite n'ayant été accordée à leur protestation, les Italiens adressent en 1933 une plainte écrite à la Commission Centrale Syndicale. Ils y résument leurs griefs et qualifient l'attitude du syndicat socialiste pendant la grève de 1932 de "politique artificielle autant que criminelle de haine fratricide." ${ }^{(29)}$

Bruno Buozzi, venu de Paris, tentera de "couvrir" ses collègues belges et expliquera aux Italiens peu convaincus - que le paiement des indemnités de grève est impossible car il n'y a pas de contrat de reciprocité signé avec les syndicats fascistes d'Italie ${ }^{(30)}$ !

\section{Après la deuxième guerre mondiale}

On sait que le patronat minier belge va faire appel massivement en 1946 à de la main-d'oeuvre italienne.

La thèse patronale est qu'il $y$ a un manque de main-d'oeuvre pour les charbonnages. En réalité, la main-d'oeuvre locale refuse d'accepter les conditions de travail et de salaires proposées. Les organisations syndicales vont adhérer à la thèse patronale et ne mettront pas en cause la nécessité de l'immigration.

C'est donc avec l'aval des syndicats belges que la grande déportation de travailleurs pour les mines est entamée.

La force des communistes en Italie fait cependant craindre qu'ils " $n$ 'importent" en Belgique leurs convictions. Un tri politique est donc prévu à l'origine (mais l'Italie proteste) et la surveillance idéologique se fait dès les convois ferroviaires pour faire déceler par la Sûreté belge les "agitateurs".

Les Italiens convaincus de communisme sont expulsés de Belgique ${ }^{(31)}$ et le patronat craint beaucoup que leurs idées ne "contaminent" leurs compatriotes via le syndicat unique qui regroupe 
socialistes et communistes.

C'est dans cette atmosphère qu'il faut resituer l'accord signé dès 1946 entre la CSC et l'Association catholique des travailleurs italiens (ACLI) pour éviter que les Italiens n'adhèrent au syndicat de gauche. A cette occasion naît également l'idée d'un journal anticommuniste (Sole d'Italia) destiné aux ouvriers italiens, dont le bilan, rapidement déficitaire, sera comblé par des fonds patronaux ${ }^{(32)}$. Le premier directeur de ce journal se glorifiait d'avoir en six mois fait disparaître ses deux concurrents de gauche, dont les rédacteurs avaient été expulsés...

L'adhésion au syndicat chrétien est encouragée par les prêtres italiens qui accueillent les immigrés italiens à leur arrivée. La CSC trouve en eux une occasion unique de renforcer ses effectifs dans un secteur (les mines) et une région (la Wallonie essentiellement) où elle est minoritaire ${ }^{(33)}$.

La syndicalisation n'a cependant pas très bonne presse parmi les Italiens. Les immigrés présents avant-guerre ont transmis aux nouveaux arrivés la mémoire de la "trahison" dont ilsont été victimes de la part des syndicats belges à l'occasion des grèves de l'entre-deux-guerres.

En 1950, le rapport moral et financier de Dehnel au Congrès ordinaire de la Centrale Syndicale des Travailleurs des mines de Belgique (15 au 17 avril) relevait :

"Les syndicats sont très peu en vogue parmi les anciens immigrés. Ils soulignent constamment que, pendant la période de grèves en 1932 et 1936, ils n'ont pas reçu les indemnités que recevaient leurs camarades belges, bien que membres de la Centrale des Mineurs. Il est très difficile de remplir les lacunes de la propagande d'avant-guerre ... Les anciens immigrés déconseillent aux jeunes l'affiliation ..." (34)

La FGTB est encore interdite d'accès dans les baraques d'étrangers en 1957 , ce qui entrave naturellement un recrutement éventuel, mais la volonté des socialistes belges de faire adhérer les Italiens n'est pas évidente dans l'après-guerre.

Le syndicat socialiste se révèle incapable de procéder à une analyse des causes économiques internes et externes de l'immigration et se révèle incapable d'entendre - malgré certaines bonnes volontés - les "autres" (colonisés du Congo, immigrés ...) avec leurs revendications propres. Malgré cela, lorsqu'une grève est déclenchée, dès 1952 les ouvriers étrangers "suivent" ${ }^{\text {(35) }}$.

Les Italiens de gauche, pour qui le syndicalisme est une chance unique d'expression sociale et politique (et bien qu'ils aient gardé des structures syndicales propres, la CGIL, syndicat communiste, est implantée via son service d'assistance de l'INCA) choisissent finalement de militer à la FGTB "malgré elle". Bien que beaucoup plus souvent communistes que socialistes, ils font le choix de militer à la FGTB pour maintenir l'unité syndicale, plutôt que de créer une fraction syndicale radicale rivale.

Dans les secteurs ou régions où leur adhésion est massive, elle va être mal perçue comme une manière d'investir ou noyauter le syndicat.

Le syndicat socialiste étant - en Wallonie surtout - une structure quasi-officielle de la société, il reproduit les comportements de la société et évacue les "marginaux".

Or les marginaux par leur origine géographique ont aussi une culture politique différente. Dans le cas des Italiens, des juifs, des Espagnols, des Portugais et dans une certaine mesure des Turcs, cette culture politique différente est aussi une culture politique plus radicale et écarter les "gauchistes" revient souvent à écarter les étrangers.

La compétition entre les deux grands syndicats implique depuis les années ' 60 qu'ils courtisent les étrangers pour les faire adhérer. On les "accueille" (!) dans les syndicats, on leur fournit les services auxquels ils ont recours.

Mais le racisme surgit à l'intérieur du syndicat au moment même où les étrangers y émergent. On refuse alors de les entendre et d'intégrer leur façon de revendiquer, de négocier, de faire la grève, d'élire des délégués. On refuse leurs revendications propres, on préfère les représenter qu'accep- 


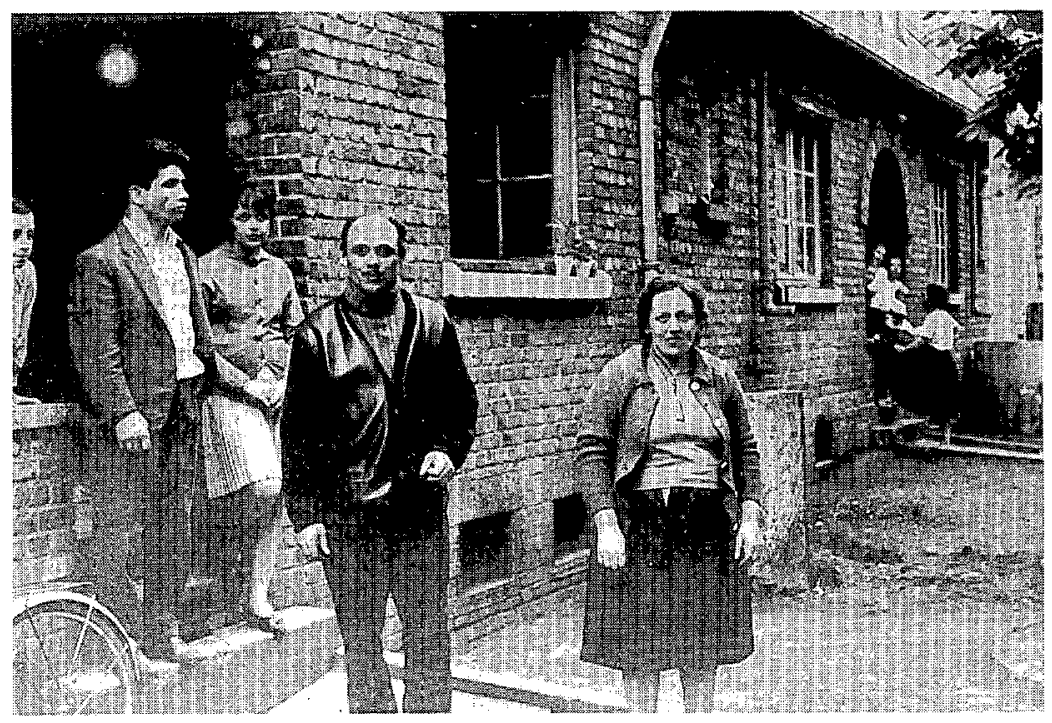

Italianen voor bun woning in Limburg, 1966

ter qu'ils se représentent ou qu'ils représentent les autochtones. L'intégration n'est comprise qu'à sens unique. Dans les deux syndicats, les immigrés sont sous-représentés tant au niveau des candidats aux élections syndicales qu'au niveau des élus ${ }^{(36)}$.

Avec des variantes (l'internationalisme prolétarien ou la charité chrétienne), malgré un discours officiel pleinement rassurant à ce sujet et quelques noms - toujours les mêmes - mis en avant comme symboliques des réussites d'étrangers, les deux grands syndicats sont probablement minés par les mêmes problèmes larvés.

L'expulsion de Lugi Carapelle de la CSC enseignement a été lue par certains comme un règlement de compte à l'égard d'un gêneur, trop remuant lors des grèves de 1990, et "macaroni" de surcroît ${ }^{(37)}$ !

A la FGTB le conflit de Boël à La Louvière - qui a vu en 1992 la FGTB "débarquer" ses propres délégués syndicaux, trop radicaux mais aussi italiens et proches du PCI - a certainement été révélateur de ce racisme souterrain. A la FGTB locale on n'hésitait pas à dire que le but de l'opération était de liquider les "macaronis"...

Ceuxquisont au pouvoir, dans lesyndicat comme ailleurs, n'hésitent devant aucun argument pour s'y maintenir. De fait peu d'actions (sauf du fait du SETCA) ont été entreprises sur le terrain des entreprises par la FGTB pour contenir les débordements racistes et au secrétariat national de la FGTB ne siège aucun syndicaliste d'origine étrangère.

On continue à parler des étrangers à "accueillir" dans les syndicats, alors qu'ils y sont de droit chez eux parce que depuis plus de septante ans les étrangers ont mené ici les luttes sociales avec les autres gens d'ici.

\section{En guise de conclusion}

Le travailn'est pas en lui-même un processus d'intégration automatique mais l'action syndicale, lorsqu'elle a vocation conflictuelle, intègre les immigrants à la fois positivement (par les pratiques communes) et négativement (en les intégrant à des pratiques conflictuelles au regard de la société globale).

Tant que le syndicat est un groupe "rebelle", en conflit avec la société dominante, il est le premier espace d'expression accessible aux étrangers. Mais la situation que nous avons sous les yeux est très diverse. Devenue une des structures de notre société, le syndicat connaît, comme toute struc- 
ture humaine, des conflits entre base et sommet, radicaux et conservateurs, "Belges" et étrangers ... Comme dans toute structure humaine de pouvoir, on y trouve - entr'autres - des alcooliques et deschefs de bandes, des racistes et des sexistes, des allergiques à toute contestation de l'autorité et d'autres qui manquent d'esprit critique, des apparatchiks et des obsédés de l'anticommunisme...

Même sice n'est pas gai à constater, j'en vois aussi dans mon propre syndicat, celui auquel j'adhère de mes cotisations et de mon coeur depuis quej'ai 19 ans!

(1) Le Peuple, 20 août 1924

(2) Le Franc-Mineur, II, 1923, II, p. 4

(3) VIllième Congrès de la Conférence des Syndicats Chrétiens de Belgique, Gand, 5-6 mai 1928, p. 70.

(4) CSC, hebdomadaire consulté de 1938 (date de sa création) à 1940 .

(5) II Riscatto, 4 février 1928

(6) /I Riscatto, 19 octobre 1930

(7) I/ Riscatto, 3 août 1930

(8) Frank CAESTECKER, Het vreemdelingenbeleid in de tussenoorlogse periode, 1922-1937 RUG, Gent, mémoire en histoire, 1983.

(9) exemple : Le mouvement syndical belge, 20 mai 1929. Le 20 avril 1940 encore, un article signé Chronos (c'està-dire Alberto Jacometti) est consacré aux "Deux Italies".

(10) Idem

(11) André PLETINCKX, Le parti ouvrier belge dans la première phase de la crise économique 1930-1933, in: Revue belge d'histoire contemporaine, VIII, 1977, 1-2, pp. 258-259.

(12) cf. Pierre VAN MALDERE, L'émigration italienne en Belgique. De qui se moque-t-on?, in: Le Peuple, 9 octobre $1929, p .1$, où le responsable syndical dénonce le recrutement d'ouvriers-peintres en Italie à des salaires beaucoup plus bas que ceux en vigueur en Belgique. Selon lui, il s'agit d'une manoeuvre pour avilir les salaires où le gouvernement est complice du patronat.

(13) cf. Le Mouvement syndical, éditoriaux des 2 janvier 1926 et 13 mars 1927, article du 27 mars 1926, p. 91, résumant la position de la CS sur le problème de l'immigration lors du congrès spécial consacré à ce sujet le 31 janvier 1926.

(14) Voir par exemple Le problème des migrations ouvrières, Cahier n 8 - mai 1925 de la Commission syndicale; Le Mouvement syndical, 20 octobre 1931, pp. 197 à 204 (semaine sociale universitaire).

(15) Le Mouvement syndical belge, 20 octobre 1931, p 202.

(16) Idem

(17) 20 janvier 1934
(18) 20 juin 1937

(19) 20 avril 1938

(20) 20 juillet 1938

(21) 20 avril 1938, p. 115

(22) Il s'avéra en outre que Silvio Barro n'était effectivement pas digne de confiance. En 1936, il prend contact avec les autorités fascistes italiennes puis rentre au pays sans y être inquiété et reçoit au parti fasciste l'ancienneté 1925! (Archivio Centrale dello Stato-Roma désormais ACS, CPC. 12.611).

(23) Drapeau rouge, Bandiera rossa, 12/13 septembre 1926.

(24) Jos DEDOYARD, en tribune libre de L'ouvrier mineur, juillet 1931, pp. 133-134.

(25) La campagne de syndicalisation qu'on peut suivre dans la "Tribuna italiana" du Drapeau rouge, prévoit même le contrôle des adhésions effectives.

(26) Lors des grèves de 1936, un indicateur du consulat italien assure che almeno il $90 \%$ degli operai italiani occupati nelle regioni industriali belghe, hanno aderito subito allo sciopero (ACS, Polizia politica, pacco 17, C 4/2, 29 juin 1936).

(27) ACP PS, Gl, b 232, fasc. 493, rapport du 21 janvier 1930 sur la réunion du 15 janvier 1930.

(28) Lettre du comité provisoire du groupe syndical italien à la Commission Centrale Syndicale en date du 31 juillet 1933 (ACS, Polizia politica, pacco 17, C 4/2).

(29) Idem

(30) Conférence du 31 mai 1933 (ACS CPC, b 2608, A. Jacometti)

(31) Aux débuts de la guerre froide, la Sûreté expulse même des Italiens qui étaient des héros de la Résistance en Belgique, $y$ habitaient depuis des décennies et $y$ avaient été décorés (cf. A. MORELLI, La participation des émigrés italiens à la résistance belge, Rome 1983).

(32) Interview d'Umberto Stefani, fondateur du journal, le 14 avril 1978 par Francesca MARINARO (Le Sole d'Italia, un journal pour les travailleurs immigrés italiens de Belgique, ULB 1978, p. 18).

(33) Je ne crois pas, comme le disent Albert BASTENIER et Patricia TARGOSZ (Les organisations syndicales et l'immigration en Europe, Sybidi Paper 11, 1991, p. 61) que la CSC s'est plus rapidement intéressée aux immigrés "parce que la matrice culturelle de l'organisation chrétienne la prédisposait davantage à une telle implication".

(34) Cité par Vinciane TILOT, Le recours à la maind'oeuvre étrangère dans les charbonnages de Belgique (1920-1959), ULB, mémoire de licence en histoire, 1973-1974, p. 173.

(35) L'adaptation et l'intégration de la colonie italienne, in: La vie économique et sociale n 4, 1957, juillet-septembre, p. 205, article d'Henri-Jacques BUCHKREMER.

(35bis) Roger WINCKEL, Quatre cents enquêtes parmi la population italienne du Borinage. Pré-sondage pour une enquête sur l'assimilation de ces travailleurs, Institut d'études sociales de l'Etat, Mons, année académique 1952-1953, p. 120.

(36) cf. BASTENIER et TARGOSZ, Les organisations syndicales [...], p. 102.

(37) Le Soir, 3 mai 1993 